\title{
Caracterización y tipología de fincas productoras de vid para Pisco en la región Ica-Perú
}

\author{
Characterization and typology of grape producing farms for Pisco in Ica-Peru region
}

\author{
Hanna Cáceres Yparraguirre ${ }^{1 *}$, Alberto Julca Otiniano ${ }^{1}$
}

\begin{abstract}
RESUMEN
El Pisco, Bebida Bandera de Perú, se remonta a lo largo de toda la historia vitivinícola peruana y se produce en la costa de los departamentos de Lima, Ica, Arequipa, Moquegua y Tacna. Favorecidos por el clima semidesértico y las condiciones óptimas para el ciclo vegetativo de la vid, Ica concentra el mayor número de áreas donde se cultiva la uva pisquera, y la mayor producción de Pisco, representando un porcentaje importante de la economía regional. Esta actividad es bastante tradicional y por ello se realizó una investigación mixta, con el objetivo de caracterizar y tipificar las fincas productoras de vid para Pisco, con base en diez componentes. Se hizo una encuesta a los integrantes de la Asociación de Productores de Piscos y Vinos de Ica. La metodología incluyó revisión documental, observación, entrevistas y encuestas a los integrantes de la asociación. Como resultado de la tipificación, se clasifican las fincas participantes en dos grupos. El grupo uno, compuesto por dos fincas y el grupo dos, compuesto por 14. La caracterización de las fincas coincide para ambos grupos en los componentes familiar, asociatividad, tecnología, gestión, capacitación y problemática que afecta al viñedo y al Pisco. Difiere en ambos grupos en los componentes social, asociatividad, económico, recursos naturales. Esta información en detalle resulta útil para los productores de Pisco, de uva, de plantas de vid, para el comercio, para el Estado y comunidad académica en general.
\end{abstract}

Palabras clave: aguardiente, pequeños agricultores, Perú, viñedos, Vitis vinífera

\begin{abstract}
Pisco, Drink Flag of Peru, goes back along all the Peruvian vitivinícola history and takes place in the coast of the departments of Lima, Ica, Arequipa, Moquegua and Tacna. Favored by the semi-desert climate and the optimum conditions for the vine's vegetative cycle; Ica, concentrates the largest number of areas where pisco grapes are grown, and the largest production of Pisco, representing a significant percentage of the regional economy. This activity is quite traditional and therefore a mixed investigation was carried out, with the objective of characterizing and typifying the vine-producing farms for Pisco, based on ten components, conducting a survey of the members of the Association of Pisco and Wine Producers. of Ica. The methodology included documentary review, observation, interviews and surveys to the members of the association. The result of the typing, groups the participating farms into two groups. Group one, composed of two farms and group two, composed of 14 farms. The characterization of the farms coincides for both groups in the familiar components, associativity, technology, management, training and problems that occur in the vineyard and Pisco. It differs in both groups in the social, associative, economic, and natural resources components. This information in detail turns out to be useful, for the producers of Pisco, of grape, of vine plants, for commerce, for the state and academic community in general.

Key words: aguardiente, small farmers, Peru, vineyards, Vitis vinífera
\end{abstract}

\section{Introducción}

Pisco es el producto obtenido exclusivamente por destilación de mostos frescos de "uvas pisqueras" recientemente fermentadas, utilizando métodos que mantengan los principios tradicionales de calidad. Se produce en la costa de los departamentos de
Lima, Ica, Arequipa, Moquegua y los Valles de Locumba, Sama y Caplina del departamento de Tacna (Consejo Regulador del Pisco, 2012; Cacho et al., 2013, CONAPISCO, 2013). Es considerado la Bebida Bandera del Perú, por lo que posee gran importancia cultural y económica. En el año 1991, el Estado peruano reconoció oficialmente al

1 Universidad Nacional Agraria La Molina (UNALM). Lima, Perú.

* Autor por correspondencia: hannacaceres@gmail.com

Fecha de Recepción: 30 mayo, 2018.

Fecha de Aceptación: 21 agosto, 2018.

DOI: http://dx.doi.org/10.4067/S0718-34292018005001002. Publicado en línea: 5-noviembre-2018. 
Pisco como Denominación de Origen Peruano a través del Decreto Supremo Nº01-91-ICTI/IND. Asimismo, en el año 2006, la Organización Mundial de Propiedad Intelectual (OMPI) reconoció al Pisco como un producto originario de Perú (Álvarez et al., 2010). Esta bebida se produce a partir de variedades de uvas criollas y algunas variedades introducidas en el Perú desde las Islas Canarias, donde las expediciones se abastecían de agua y víveres. Entre ellas pueden nombrarse las pasas (Lacoste et al., 2010; Pszczólkowski y Lacoste, 2016). A partir de 1532, en el Perú se fundaron villas y ciudades, y poco después se inicia el cultivo de los llamados "frutos de Castilla", es decir, trigo, caña de azúcar, arroz, vid, olivos, cítricos y muchas plantas más (Huertas, 2004). De este modo, los españoles se percataron de las condiciones del clima y de los terrenos llanos, así como de las zonas más bajas de los valles costeros, que se prestaban muy bien para el cultivo de las vides (Soldi, 2006). Así tenemos, a $300 \mathrm{~km}$ al sur de la capital de Perú, la región de Ica, caracterizada por su clima cálido, sus plantaciones de uva y sus bodegas de Pisco y vino. Se destina una superficie total de 7.662 ha para su cultivo, de las cuales 3.164 ha son utilizadas para la elaboración de Pisco y vino. Esta actividad representa un porcentaje importante de la economía regional, pues la producción pisquera de Ica constituye el $60 \%$ - $65 \%$ de la producción nacional de Pisco. La actividad vitivinícola es bastante tradicional en dicha región. Sin embargo, a partir del año 2002, coincidiendo con la instalación del Centro de Innovación Tecnológica Vitivinícola (CITEvid), en la actualidad CITE agroindustrial, este sector vitivinícola presenta un fuerte dinamismo que se refleja en el posicionamiento del mismo. En efecto los CITE son centros de servicios y transferencia tecnológica para las pymes del espacio geográfico en el cual se ubican. Estos centros son auspiciados por el Ministerio de Producción, y pueden ser públicos o privados (Álvarez et al., 2010). Por otro lado, en el tema pisquero actualmente existe una asociación muy conocida denominada Asociación de Productores de Piscos y Vinos de Ica (APROPICA), y resulta importante caracterizarla y tipificarla para identificar los principales puntos críticos que pudieran afectar su perpetuidad. La caracterización tiene los siguientes objetivos: a) conseguir información técnica de referencia sobre las prácticas productivas y la productividad en el lugar de estudio; b) entender el proceso de toma de decisión de los productores en relación con el funcionamiento de sus sistemas de producción; y c) identificar los principales factores limitantes (físicos, biológicos y económicos) y las posibilidades de generar alternativas para los sistemas caracterizados (Santistevan, 2016). Las experiencias indican que la selección de áreas para desarrollar proyectos de generación de tecnologías tiene usualmente un alto grado de heterogeneidad. Esto como resultado no solamente de las condiciones ecológicas, sino también de aspectos económicos de la estructura de producción, la disponibilidad de factores de producción y la influencia de condiciones macro, como la especialización del mercado y el acceso a los servicios y la infraestructura necesaria para la producción (Escobar, 1986 citado por Tuesta, 2014). La tipificación, entre otras cosas, permite conocer la organización conceptual de la diversidad existente en la agricultura local, una lista de unidades de producción representativa y la población vinculada a los sistemas de producción (Escobar \& Berdegué 1990 citado por Tuesta, 2014). Una finca puede tenercuatrotipos básicos de procesos de producción: 1) producción agrícola (incluida la silvicultura); 2) producción pecuaria (incluye toda clase de animales); 3) procesamiento de productos; 4) transacciones entre la finca y el ambiente que la rodea (incluido todo tipo de compra, venta, comercialización e inversión) (Santistevan, 2016). Con base en los antecedentes anteriores, la presente investigación planteó la interrogante sobre la situación que prevalece en las prácticas cotidianas de las fincas de una asociación de productores representativa de Ica. En este contexto, el objetivo del presente estudio fue caracterizar y tipificar las fincas productoras de vid para Pisco, con base en los componentes familiar, social, asociatividad, actividad en la finca, económico, tecnología, gestión, capacitación, recursos naturales y problemática del viñedo y del Pisco en la principal zona productora de vid pisquera y de Pisco, lo que actualmente es Ica-Perú.

\section{Materiales y métodos}

\section{Descripción del área de estudio}

El ámbito del estudio comprendió los distritos de la provincia de Ica, ubicada en la costa central del desierto y a $300 \mathrm{~km}$ al sur de la capital del Perú (Figura 1). Su clima es típicamente árido, la duración de la insolación diaria tiene un valor medio de 7 horas. La temperatura media es de $21^{\circ} \mathrm{C}$ con una media máxima de $29^{\circ} \mathrm{C}$ y una media mínima de $14^{\circ}$ C, y la precipitación anual es de casi cero (cantidad a 
a

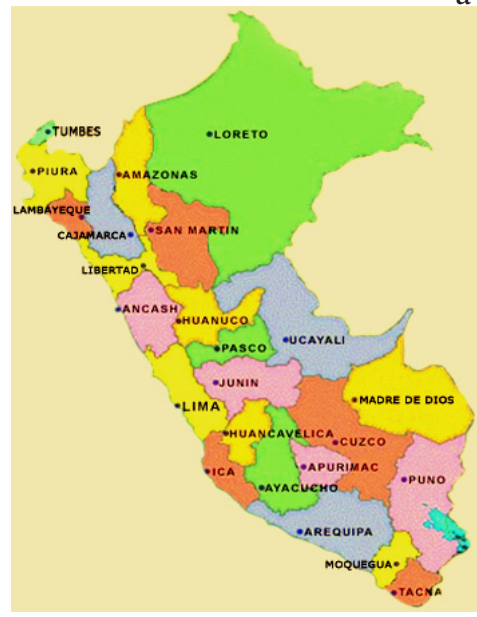

$\mathrm{b}$

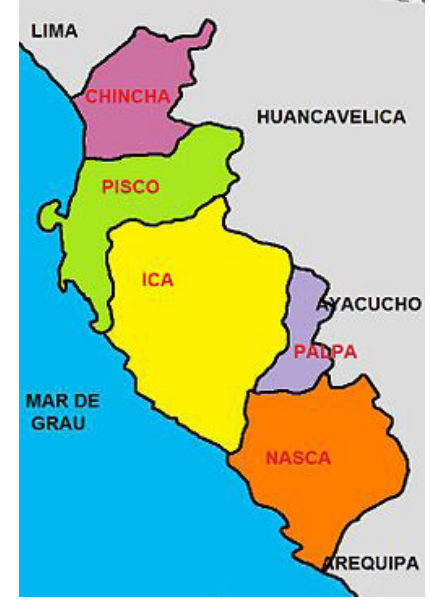

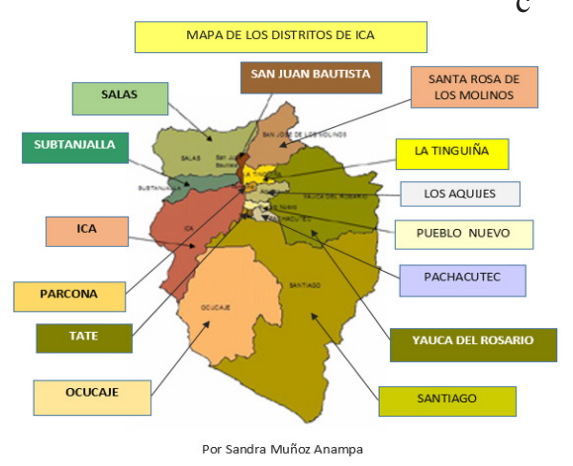

Figura 1. (a) Mapa de Perú con sus departamentos (b) mapa de Ica con sus cinco provincias (c) mapa de provincia de Ica, mostrando sus catorce distritos. Fuente GORE, ICA (2017)

\section{Metodología}

\section{Diseño de la investigación}

La investigación se realizó en las fincas productoras de vid de los integrantes de APROPICA y es de tipo descriptivo - explicativo.

\section{Diseño de la población y muestra}

La población objetivo comprendió a 53 socios, a los cuales se les invitó a participar en la investigación a través de la entrevista y llenado de encuestas. Los productores cuentan con fincas completas e incompletas que poseen producción agrícola, producción pecuaria, procesamiento de productos $\mathrm{y}$ transacciones entre la finca y el ambiente que la rodea. Los integrantes de APROPICA se caracterizan por tener la Denominación de Origen para producir Pisco y por considerarla la más representativa de esta provincia. Para definir la muestra se tomaron en cuenta dos criterios:

Criterio de inclusión: socio que se encuentre en activa participación en APROPICA en el momento de la encuesta.

Criterio de exclusión: a) Deseo de no participar en la encuesta; b) No contar con el componente producción agrícola; c) No contar con ningún componente de la finca: producción agrícola, producción pecuaria, procesamiento de productos y transacciones entre la finca y el ambiente que la rodea.
Muestra: finalmente, luego de haber pasado los criterios de inclusión y exclusión, la muestra total fue de 16 fincas.

\section{Técnicas de recolección y análisis de la información}

Las técnicas de recolección usadas fueron:

- Fuentes de información primaria y secundaria: se realizó una recopilación bibliográfica referente a la Denominación de Origen del Pisco

- Entrevistas a los expertos técnicos: con el fin de seleccionar los componentes de la encuesta y las principales variables cuantitativas y cualitativas a consultar. Los expertos técnicos fueron profesionales del CITEagroindustrial, desde el director de la institución, así como los profesionales dedicados al manejo de viñedo y a la elaboración de Pisco.

- Encuesta: constituida por diez componentes y aplicada a los 16 representantes de las fincas que pasaron los criterios de inclusión y exclusión.

- Procedimiento de análisis de datos: para determinar el agrupamiento (tipología) de fincas se realizó un análisis conglomerado, por el método Ward. Asimismo, se aplicó el Análisis de Varianza Multivariada para las variables cuantitativas y así poder determinar diferencias significativas entre los grupos encontrados, y la Prueba de Duncan para comprobar la significancia de cada variable seleccionada 
dentro del conglomerado. Para las variables cualitativas se realizaron tablas de contingencia utilizando Chi cuadrado para definir diferencias estadísticas entre grupos. También se empleó estadística descriptiva para detallar ciertas características de los grupos formados. El análisis de conglomerados se hizo utilizando las 24 variables cuantitativas de la encuesta realizada. Las variables cualitativas se convirtieron a numéricas dicotómicas $(0,1)$ para facilitar el procesamiento; los grupos identificados fueron caracterizados y diferenciados entre sí, mostrando la potencialidad, así como los limitantes de cada uno (Tuesta, et al., 2014)

\section{Resultados y discusión}

La caracterización y tipificación de las fincas productoras de vid para Pisco se ha realizado teniendo en cuenta la unidad central de análisis: finca.

\section{Caracterización de las fincas respecto a los 10 componentes en estudio}

Se describen las características de las fincas, de acuerdo a los dos grupos obtenidos en la tipificación del total de la muestra, para cada componente evaluado.

En cuanto a las variables del componente familiar, los dos grupos guardan relación con respecto a la instrucción superior que posee el responsable de la finca, que es originario de la región de Ica. Para el desarrollo de la finca en sus diversas áreas cuentan con integración familiar: la familia vive en la finca, pudiendo ser en el área agrícola, área de procesamiento o en el área de comercialización. Las viviendas que habitan son adecuadas para las condiciones climáticas de Ica y se encuentran satisfechos con la producción de su finca. No hay diferencia significativa respecto a la edad del responsable en los dos grupos de fincas: fluctúa entre 53 y 62 años. En las fincas del grupo 1, el responsable es un hombre, mientras que en las del grupo 2, un 29\% de los responsables son mujeres. En las fincas del grupo 1, el responsable dedica el $100 \%$ de su tiempo al desarrollo y éxito de estas. Entre las fincas de los grupos 1 y 2 no hay relación respecto a que han sido traspasadas de una generación a otra. Parcialmente ha sido así, pero también se observa que han sido adquiridas de otros propietarios o se ha comprado terreno donde se ha iniciado el cultivo de vid. Esta información concuerda con lo afirmado por
Álvarez et al. (2010), quienes mencionan que en el clúster vitivinícola de Ica hay una construcción de conocimiento de generación en generación, el cual es en su mayor parte tácito. En el componente social, los datos obtenidos indican que los dos grupos de fincas guardan relación en cuanto a que cerca de ellas existen instituciones de salud para un auxilio rápido ante una emergencia; hay instituciones para educar a sus hijos o para los hijos de los trabajadores y además cuentan con comisarías o reciben visitas de personal de seguridad de la municipalidad del distrito. Los caminos para llegar a la finca son adecuados, existe movilidad pública para trasladarse hasta allá y cuentan con medios de comunicación, principalmente señal de telefonía móvil, radio y televisión. En los dos grupos de fincas no guardan relación respecto a los beneficios sociales que reciben los trabajadores: solo el $100 \%$ de los trabajadores de las fincas del grupo 1 tienen esa ayuda. Existe diferencia significativa entre ambos grupos respecto al número de personas que trabajan en la finca, incluyendo trabajadores permanentes, eventuales, hombres y mujeres. Existe diferencia significativa en el número de trabajadores permanentes en los dos grupos de fincas, es decir, hay en promedio nueve trabajadores en el grupo 1 y tres en el grupo 2 . No hay diferencia significativa en el número de trabajadores eventuales y de mujeres que trabajan en los dos grupos de fincas. Estos resultados coinciden con lo que sostienen Molero et al., 2007, quienes al realizar la caracterización del sistema de producción de la uva de vino en el municipio Mara, estado Zulia - Venezuela, concluyeron que el mayor porcentaje de los trabajadores no son permanentes y hacen diferentes labores para manejo del cultivo, mientras que el menor porcentaje corresponde a los trabajadores que están fijos o permanentes en la finca encargados de su producción y administración. En el componente asociatividad los datos obtenidos indican que los dos grupos de fincas coinciden en que están satisfechos de trabajar en asociatividad en APROPICA, y esta les genera confianza. Estos datos guardan relación con lo presentado por Molero et al., 2007, donde se menciona como una oportunidad la existencia de una Asociación de Viticultores y una Cooperativa en la zona de estudio. Destacan que eso constituye una oportunidad para el desarrollo de este rubro, ya que los productores pueden organizarse para mejorar tanto la producción como la comercialización del producto y conseguir precios bajos para la compra de los insumos y agroquímicos. Por otra parte, también se ha podido evidenciar que para las fincas del grupo 1 se menciona 
que en APROPICA no hay colaboración entre los socios y existe competencia entre ellos. Al respecto, Álvarez et al. (2010) afirman que conseguir el posicionamiento implica alcanzar logros y objetivos como la sobrevivencia entre competidores. Así vemos que a partir del año 2001 se han dado notables desarrollos en el tema de las mejoras tecnológicas y organizacionales entre las empresas del clúster vitivinícolade Ica, evidenciándose su posicionamiento. El clúster compite con empresas y también con clústeres de otras regiones y de otras latitudes. En particular, hay una fuerte competencia con productores chilenos que está llevando al reconocimiento de la Denominación de Origen Pisco a favor del Perú y que significó que el 8 de febrero del año 2003 sea declarado en Chile como Día de la Piscola, en tanto que el mismo día también fue declarado en el Perú como Día Nacional del Pisco Sour. Esta fuerte competencia ha significado un mayor interés en las organizaciones gubernamentales y de la academia en insertarse y apoyar a las empresas del conglomerado. En el componente actividad en la finca, los datos obtenidos indican que, para los dos grupos de fincas, se guarda relación en contar con área vitícola, área de procesamiento, área pecuaria y área de comercialización. Esta a su vez ofrece turismo. No hay relación entre los dos grupos de fincas con respecto a la oferta de gastronomía. El 50\% de las fincas del grupo 1 brindan ese valor adicional al negocio, mientras que en las del grupo 2 solo el $21 \%$ ofrece ese servicio. Existe diferencia significativa en el área total de las fincas, en el área asignada para el cultivo de vid, en el área destinada para el procesamiento del Pisco, en el área utilizada para otros cultivos y en la edad promedio de los viñedos en los dos grupos. Las fincas del grupo 1 tienen mayor extensión en las áreas y sus viñedos son más jóvenes (31 ha y 4 años en promedio) que los de las del grupo 2 (4 ha y 22 años en promedio). Molero et al. (2007) sostienen en su investigación que las cuatro fincas en estudio difieren en el número de hectáreas y que tienen viñedos de 23 ha; 6 ha; 5 ha y 7 ha. También manifiestan que la edad de los viñedos no es la misma, ya que existen viñedos de 20, 23, 50 y 15 años. Es decir, las plantas tienen más años de instaladas que las plantas del presente estudio. En el componente económico, los datos obtenidos indican que, en los dos grupos de fincas, el 50\% de su producción de uva es suficiente para elaborar Pisco, pero existe otro 50\% de fincas que necesitan comprar uva para lograr la producción que demandan sus clientes. Además, se guarda relación entre los dos grupos en que venden Pisco en la finca, en mercado local y nacional. Estos datos coinciden parcialmente con la investigación realizada por Álvarez et al. (2010), donde mencionan que en el clúster vitivinícola de Ica, el principal mercado de venta es regional, nacional e internacional. Por otro lado, los propietarios de estas fincas realizan otras actividades adicionales de ingreso económico para el sustento de la familia; consideran que la finca es rentable; cuentan con presupuesto para pagar capacitación; tienen acceso a crédito; realizan pago por derecho de uso de agua: creen que dedicarse a este sector y al Pisco les da tranquilidad para vivir o subsistir; todos producen Pisco como principal producto y en segundo lugar producen mistela, que también recibe el nombre de vino dulce o vino de misa. Estos datos coinciden con la investigación realizada por Álvarez et al. (2010), donde mencionan que la producción es diversificada en el clúster vitivinícola de Ica y los principales productos son vino y Pisco. Existe diferencia significativa entre los dos grupos de fincas respecto a la producción anual de vid (TM/Ha) en el 2016. En las fincas del grupo 1 se obtienen $25 \mathrm{TM} / \mathrm{Ha}$ y en las del grupo 2 se logran 13 TM/Ha. No existe diferencia significativa entre los dos grupos de fincas en el número de variedades de vid pisquera que cultivan. Generalmente los viticultores tienen en promedio de tres a dos variedades de uvas pisqueras. Siempre cultivan la variedad Quebranta, seguida de la Torontel, Moscatel o Italia. Tampoco existe diferencia significativa en la producción de litros de Pisco en las fincas de ambos grupos, ni en el número de $\mathrm{kg}$ de vid para producir 1 litro de Pisco, que en promedio para los dos es de 7 $\mathrm{kg}$. Esto coincide con lo mencionado por Álvarez et al. (2010), quienes estiman un rendimiento en la producción de Pisco de $7 \mathrm{~kg}$ de uva pisquera por litro de Pisco. Tampoco existe diferencia significativa en el ingreso económico en S/; en el costo de producción de $1 \mathrm{~kg}$ de uva; en el precio en $\mathrm{S} /$ al que venden la botella de Pisco de 0,75 l; en el costo en S/ de inversión en marketing; en el número de otros cultivos que sacan a la venta. En el componente gestión, los datos obtenidos indican que los dos grupos guardan relación respecto a que los productores consideran que desde que se han iniciado en la finca han evolucionado; participan en conjunto en ferias para comercializar y promocionar sus productos; se han presentado en concursos regionales y nacionales en los últimos cinco años; consideran que sí saben vender Pisco y sus fincas cuentan con título de propiedad. No guardan relación 
los dos grupos, respecto a que en los últimos cinco años han obtenido medallas por la calidad de su producto. Solo las fincas del grupo 1, el 100\%, han logrado alguna medalla, generando mayor comercialización y estatus de su producto respecto a los otros productos que no han sido favorecidos. Los concursos regionales, nacionales e internacionales de Pisco son una forma de interacción entre productores, consumidores y catadores. Estos concursos son realizados con cierta frecuencia y tienen mucha importancia. El concurso nacional de Pisco se celebra una vez al año y es organizado por la Comisión Nacional del Pisco (CONAPISCO). Álvarez et al. (2010). En el componente capacitación, sí guardan relación los datos obtenidos para los dos grupos respecto a la participación en capacitaciones brindadas por diferentes entidades y a que aplican en sus fincas los conocimientos adquiridos. Al respecto, Álvarez et al. (2010) manifiestan que el CITE agroindustrial ex CITEvid fue una de las instituciones que transfirieron conocimientos a los productores de vid en el manejo del agua y en la elaboración de Pisco. En el componente recursos naturales, sí guardan relación los dos grupos de fincas respecto a la aptitud del suelo y al agua para la viticultura. Un sistema es sustentable si las prácticas mantienen o mejoran la vida en el suelo, si el manejo de las parcelas dedicadas al consumo es adecuado para la conservación de los recursos, sobre todo de la vida del suelo (Sarandon et al. 2006). Por otro lado, ninguno de los dos grupos de fincas tiene área de conservación o de corredores biológicos y tampoco disponen de algún espacio con flores para albergar insectos benéficos, polinizadores, etc. Estudios de caso en viñedo orgánico en el Norte de California por Nicholls (2001) sugieren que es posible restaurar los controladores naturales en los agroecosistemas a través de la diversificación vegetal en cualquier área del mundo mediterráneo. La biodiversidad es importante para la regulación del sistema ya que, entre otras funciones, proporciona hábitat y nichos ecológicos para los enemigos naturales. A la vez la diversidad está relacionada con la conciencia y conocimiento ecológico. La poca conciencia y conocimiento ecológico repercute claramente en el deterioro de los recursos debido al efecto sobre la biodiversidad, la biología del suelo y el riesgo de erosión (Sarandón et al. 2006). Tampoco guardan relación los dos grupos de fincas respecto a la calidad del suelo en cuanto al drenaje. Existe diferencia significativa entre ambos grupos con relación a las variables número de riegos que realizan por campaña y número de riegos que realizan con agua temporal. En el componente problemática en el viñedo, los datos obtenidos indican que los dos grupos de fincas coinciden en que los contratiempos en el viñedo son por sequía. Ello concuerda con la investigación realizada por Álvarez et al. (2010), donde mencionan que hay gran preocupación en Ica por la escasez del agua, que podría tornar al valle en una zona seca en 15 años, haciendo colapsar todo el esfuerzo agroexportador desplegado en esta región. Se requieren por lo menos $10 \mathrm{~m}^{3} / \mathrm{s}$ adicionales para satisfacer la demanda del agua. Esto es particularmente sensible en el sector de Río Seco - Pampa de Villacurí, donde toda el agua utilizada proviene de pozos y pese a prohibirse el perforado de nuevos pozos se estaría agotando la napa freática. Ante esta situación han sido planteadas algunas alternativas como la de la represa Polvareda para represar el río Pisco y de ahí derivar 10 $\mathrm{m}^{3} / \mathrm{s}$ hacia el sector de Río Seco-Pampa de Villacurí. Otra alternativa sería canalizar las aguas de la cuenca Incahuasi del río Pampas en Huancavelica hacia la laguna de Choclococha, de donde se suministraría agua desde la parte alta de Ica. Esta propuesta se podría combinar con un proyecto hidroeléctrico. También se ha planteado hacer partícipe a Huancavelica del boom agroexportador de Ica a través de un canon hídrico. Adicionalmente, consideran que el problema en el viñedo es por plagas y enfermedades. Esta información coincide con lo publicado por Morelo et al. (2007), quienes indican que la mayoría de las variedades de vid son muy susceptibles a ser atacadas por enfermedades principalmente fungosas (oídium: Uncinula necátor; Mildíu lanoso: Plasmopara vitícola; y podredumbre: Botrytis cinerea). También por trips, ácaros, comején, avispas, pájaros y malezas, cuya incidencia guarda relación con la precipitación, humedad relativa y temperatura dominante. El control de estas plagas se hace mediante la aplicación de plaguicidas, insecticidas, acaricidas y repelentes para el caso de pájaros. En los viñedos de este estudio, se ha observado el daño por filoxera (Dactylosphaeria vitifoliae) en su estado gallícola y radicícola. Esta plaga es continua en los viñedos, ya que los agricultores utilizan plantas francas y no emplean patrones americanos resistentes al insecto. También se observan problemas por chanchito blanco (Planococcus sp.), que va aumentando su incidencia. Por otro lado, cada vez es más frecuente reportar daños en los viñedos por presencia de nematodos y daño a las yemas por presencia de ácaros. También son una fuerte plaga las aves en época de cosecha. Se 
suman a la problemática del viñedo la falta de presupuesto de los productores y la delincuencia en aumento. Estos resultados coinciden con lo que sostienen Molero et al. (2007), quienes presentan como amenaza al cultivo, el aumento de los precios de los insumos y agroquímicos, que se refleja en el precio de sus cosechas y que conduce al deterioro de la calidad de la fruta. A ello se agrega la inseguridad presente en la zona, que acarrea graves problemas a los productores, quienes tienen que tomar medidas estrictas para cuidar sus cosechas y su integridad física, así como la de su familia y sus trabajadores. $\mathrm{Al}$ mismo tiempo, consideran que la falta de mano de obra cada vez va en aumento en Ica. Estos datos no guardan relación con lo que sostienen Molero et al. (2007), quienes mencionan como gran fortaleza del cultivo la disponibilidad y calidad de mano de obra proveniente generalmente de los pobladores de la zona que se han capacitado en el transcurso de los años, perfeccionando las labores. En Ica sucede que las áreas para el cultivo de uva de mesa u otros cultivos de agroexportación han crecido y por lo tanto también son demandantes de mano de obra. Al ser grandes empresas otorgan los beneficios sociales de ley a los trabajadores, que prefieren trabajar con ellos y no con los pequeños agricultores. Respecto a la problemática del Pisco se mencionan varias causas, entre ellas, adulteración de Pisco con otros alcoholes y la producción de aguardiente a base de otras variedades de vid no registradas en el Reglamento de Denominación de Origen. En este contexto, el MINCETUR (2004) señala que para que el Pisco pueda seguir consolidándose en el mercado internacional, necesita mejorar sus ventajas competitivas, es decir, debe potenciar el sector a través de inversión en tecnología, capacitación a los productores, estandarización de los productos y niveles de calidad, fortalecimiento del posicionamiento de la marca país y reducción de la informalidad y de la falsificación de productos. Esto permitiría mejorar el impacto en el ambiente comercial, donde se mueve el negocio del Pisco. $\mathrm{Al}$ respecto, se necesita que la empresa, comunidad académica y Estado realicen mayor investigación y desarrollo de nuevos productos usando la uva de descarte y así disminuir las perdidas de materia prima. También se indican como otros factores que inciden en la problemática del Pisco, no ser considerado prioritario para la alimentación, los altos impuestos asignados, la falta de apoyo a los productores y la existencia de productores informales.

\section{Tipificación de fincas productoras de vid para Pisco en Ica, Perú, en función de las variables cuantitativas}

El método de agrupamiento jerárquico de Ward y la distancia euclidiana al cuadrado permitió identificar dos grupos de fincas a una distancia de 117,54 (Figura 2). El primer grupo está formado por dos fincas (13\%), las cuales reciben el código C - J. Estas fincas se caracterizan por contar con área agrícola, área de procesamiento, área de comercialización y una de ellas dispone de área pecuaria. La parte vitícola cuenta en promedio con 31 ha y es el área más grande de la finca. En promedio, 13 ha se destinan para otros cultivos. La edad promedio del viñedo es de 4 años. En estas fincas se da mayor producción de uva que en las del segundo grupo, en promedio $25 \mathrm{TM} / \mathrm{Ha}$, lo que coincide con la investigación realizada por Álvarez et al. (2010), donde mencionan que la uva pisquera tiene una producción anual promedio de $25 \mathrm{TM} / \mathrm{Ha}$. La agricultura que practican es convencional. En estas fincas el riego es exclusivamente tecnificado, es decir, riego por goteo; realizan fertilización química, utilizan herbicidas, tractor para labrar el suelo, controlan las plagas con agroquímicos en su totalidad, sus viñedos cuentan con sistema de conducción, el suelo y el agua son aptos para la viticultura. Una de las fincas de este grupo implementa buenas prácticas agrícolas. Respecto al área de procesamiento, en estas fincas se produce mayor cantidad de Pisco que en el otro grupo: 39,500 litros en promedio. Asimismo, el área pecuaria de una de estas fincas es muy pequeña y se dedican a la crianza de animales menores como aves de corral. Finalmente, en el área de ventas se ofrece turismo y gastronomía a los visitantes nacionales e internacionales. Son las fincas que más invierten en marketing y hacen más uso de redes sociales. El segundo grupo está formado por 14 fincas (87\%), las cuales reciben el código A, B, D, E, F, G, H, I, $\mathrm{K}, \mathrm{L}, \mathrm{M}, \mathrm{N}, \mathrm{O}, \mathrm{P}$. Estas fincas se caracterizan por contar en $100 \%$ con área agrícola y parcialmente con área pecuaria, área de procesamiento y área de comercialización. La parte vitícola dispone en promedio de 4 ha y es el área más grande de la finca. En promedio 1 ha se destina para otros cultivos. $\mathrm{La}$ edad promedio de los viñedos de estas fincas es de 22 años. Se produce en promedio $13 \mathrm{TM} / \mathrm{Ha}$ de uva y la agricultura que practican es en su mayoría convencional, pero también se dedican a la agricultura sustentable. En estas fincas el riego en su mayoría es por gravedad, y parcialmente realizan fertilización 
química y utilizan herbicidas. En su mayoría utilizan animales para labrar el suelo. La mayoría de estas fincas controlan las plagas con agroquímicos. Sus viñedos cuentan con sistema de conducción con postes de madera o de cemento, el suelo y el agua son aptos para la viticultura. Respecto al área de procesamiento, se produce menor cantidad de Pisco, en promedio 21 mil litros. El área pecuaria es muy pequeña al igual que la de las fincas del primer grupo y se dedican a la crianza de animales menores como aves de corral. En el área de ventas se ofrece turismo y en menor cantidad de fincas se brinda gastronomía a los visitantes nacionales e internacionales. Son las fincas que menos invierten en marketing y menos usan las redes sociales.

\section{Conclusiones}

Este trabajo representa la primera caracterización y tipificación de fincas de vid para Pisco en la región Ica-Perú, utilizando 10 componentes, lo que es muy

\section{Ward}

Correlación cofenética $=0,569 ;$ Distancia:Euclidea^2

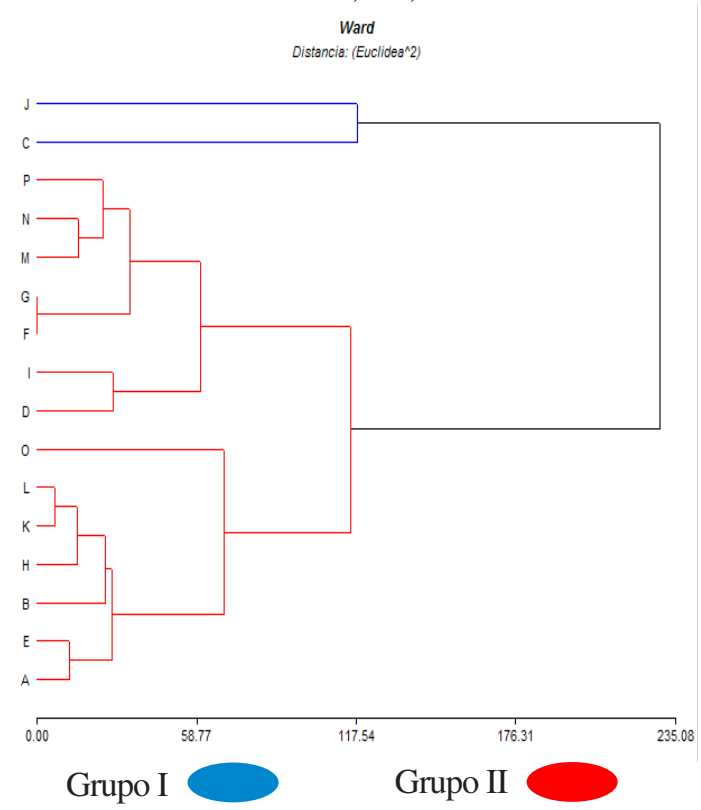

Figura 2. Dendograma basado en la distancia Euclídea al cuadrado y método de Ward para 16 fincas utilizando variables cuantitativas obtenidas de una encuesta realizada. útil para los productores de Pisco, de uva, de plantas de vid, para la empresa, para el Estado y comunidad académica en general. $\mathrm{Al}$ respecto se detalla lo siguiente:

\section{- Caracterización de las fincas respecto a los 10 componentes en estudio}

El primer grupo está formado por dos fincas que se caracterizan por contar con área agrícola con más de 45 ha, donde la mayor superficie es para la parte vitícola. También disponen de área de procesamiento, una pequeña área pecuaria, con área de comercialización, y ofrecen turismo y gastronomía. Todas practican la agricultura convencional y presentan mayor producción de uva. En este grupo de fincas sus trabajadores reciben beneficios sociales. $\mathrm{La}$ edad promedio de los viñedos es de 4 años, el costo de producción de un $\mathrm{kg}$ de uva es $\mathrm{S} / 0,50$ y el precio al que venden una botella de Pisco de 0,75 litros es S/ 28,00 en promedio. Son las fincas que producen más litros de Pisco en la campaña 2016. El 100\% considera que el mayor problema del Pisco es el aguardiente elaborado de otros variedades de vid no registradas en el reglamento de Denominación de origen, dañando la calidad e imagen del producto que esas fincas elaboran y a nivel nacional.

El segundo grupo está formado por fincas que se caracterizan por contar con área agrícola, con un promedio de 5,5 ha, donde la mayor superficie es para la parte vitícola. También poseen área de procesamiento, una pequeña área pecuaria, con área de comercialización, y ofrecen turismo y gastronomía. Las fincas de este grupo producen en promedio más de 21 mil litros de Pisco y venden la botella de 0,75 litros en $\mathrm{S} / 22,00$. Consideran que la amenaza del viñedo es la sequía y las amenazas del Pisco son otros aguardiente y los altos impuestos asignados.

\section{- Tipificación de las fincas productoras de vid para pisco en Ica, Perú}

Las 24 variables cuantitativas han permitido clasificar las 16 fincas en dos grupos. El primer grupo está formado por 2 fincas (13\%), las cuales reciben el código $\mathrm{C}$ - J. El segundo grupo está compuesto por 14 fincas (87\%), las cuales reciben el código A, B, D, E, F, G, H, I, K, L, M, N, O, P. 


\section{Literatura citada}

Abreu, M.; Bannon, P.

1993. Dynamics of the South American coastal desert. $J$. Atmos. Sci., 50: 2952-2964.

Álvarez, J.; Dlpolitto, C.; de Aguiar, E.

2010. Estudio de caso innovador: Pisco Payet. En: CIESFINCyT Innovación empresarial y comportamiento tecnológico sectorial. Lima, Perú. Pp. 553-608.

Cacho, J.; Moncayo, L.; Palma, J.; Ferreira, V.; Culleré, L. 2013. The Impact of Grape Variety on the Aromatic Chemical Composition of Non-Aromatic Peruvian Pisco. Food Research International, 54: 373-381.

CONAPISCO.

2013. Historia del Pisco Peruano. Comisión Nacional del Pisco. Disponible en http://www.conapisco.org.pe/elpisco.html Consultado: 30 de mayo 2018.

Consejo Regulador del Pisco.

2012. Reglamento de la Denominación de Origen Pisco 2011. INDECOPI. Perú. pp.29.

Huertas, L.

2004. Historia de la Producción de Vinos y Piscos en el Perú. Revista Universum, 19 (2): 44-61.

Lacoste, P.; Muñoz, J.G.; Castro, A.

2010. Variedades de uva en Chile y Argentina (1550-1850). Genealogía del Torrontés. Mundo Agrario, 10 (20): 1-36.

MINCETUR,

2004. Plan operativo del producto pisco. PRX:Ica. Ministerio de Comercio Exterior y Turismo. Pp.11.

Molero, T.; Guerrero, R; Martínez, E.

2007. Caracterización del sistema de producción de uva de vino en el municipio Mara, estado Zulia. Venezuela. Rev. Fac. Agron. (LUZ). 2007, 24: 343-366.
Nichols, C.

2001. Capítulo 29. Manipulando la biodiversidad vegetal para incrementar el control biológico de insectos plaga: un estudio de caso de un viñedo orgánico en el Norte de California. En: Sarandón, s. (Ed.). Agroecología, el camino hacia la agricultura sustentable. Ed. Científicas Americanas. Pp. 495-513.

Pszczólkowski, P.; Lacoste, P.

2016. Variedades criollas, una oportunidad para el pisco de Chile. Rev. FCA Uncuyo, 48 (1): 239-251.

Santistevan, M.

2016. Sustentabilidad de las fincas cafetaleras en Jipijapa (Manabí, -Ecuador). Revista Saber y Hacer, 3 (1): 23-35.

Sarandón, S.; Zuluaga, M.; Cieza, R.; Gómez, C.; Janjetic,

L.; Negrete, E.

2006. Evaluación de la sustentabilidad de sistemas agrícolas de fincas de misiones, Argentina, mediante el uso de indicadores. Agroecología, 1: 19-28.

Soldi, A.

2006. La Vid y el Vino en la Costa Central del Perú, siglos XVI y XVII. Rev. Universum, 21(2): 42-61.

Tuesta, O.; Julca, A.; Borjas, R.; Rodríguez, P.; Santistevan, M. 2014. Tipología de fincas cacaoteras en la subcuenca media del río Huayabamba. Distrito de Huicungo (San Martín - Perú). Ecología Aplicada, 13 (2): 71-78.

Yzarra W.; Sanabria J.; Cáceres H.; Solis O.; Lhomme J.P. 2015. Impact of climate change on some grapevine varieties grown in Peru for pisco production. J. Int. Sci. Vigne Vin. 49, 103-112. 
